\title{
Repeated bond traversal probabilities for the simple random walk
}

\author{
T. Antal ${ }^{1}$, H.J. Hilhorst ${ }^{2}$, and R.K.P. Zia ${ }^{3}$ \\ ${ }^{1}$ Département de Physique Théorique, \\ Université de Genève, CH 1211 Genève 4, Switzerland \\ ${ }^{2}$ Laboratoire de Physique Théorique, Bâtiment 210, \\ Université de Paris-Sud, 91405 Orsay cedex, France \\ ${ }^{3}$ Center for Stochastic Processes in Science and Engineering, \\ Department of Physics, \\ Virginia Polytechnic Institute and State University, \\ Blacksburg, Virginia 24061, USA
}

\begin{abstract}
We consider the average number $B_{m}(t)$ of bonds traversed exactly $m$ times by a $t$ step simple random walk. We determine $B_{m}(t)$ explicitly in the scaling limit $t \rightarrow \infty$ with $\mathrm{m} / \sqrt{t}$ fixed in dimension $d=1$ and $m / \log t$ fixed in dimension $d=2$. The scaling function is an erfc in $d=1$ and an exponential in $d=2$.
\end{abstract}

PACS 05.40.Fb

\section{INTRODUCTION}

The simple random walk is a venerable problem, finding its applications in many areas of statistical physics. Despite its long history, novel aspects continue to surface. Motivated by recent experiments on gas transport properties in polycarbonate films, in which polymers are found in a glassy state, we are led to the the following question. Suppose the polymers are modeled by the usual (non-interacting) random walks on a lattice, how many "monomers" can we expect on each bond? Clearly, the answer will depend on both the length and the density of the polymers. Not surprisingly, it is a simple step once we know the distribution of bond-traverses of a single polymer (or random walker). Remarkably, this distribution is not, to the best of our knowledge, in the literature. This note will be devoted to our findings of such a distribution.

Let a simple random walk start at the origin of a $d$-dimensional hypercubic lattice. We wish to know the average number of bonds $B_{m}(t)$ that it has traversed exactly $m$ times. This quantity satisfies

$$
\sum_{m=1}^{\infty} B_{m}(t)=B(t), \quad \sum_{m=1}^{\infty} m B_{m}(t)=t
$$

where $B(t)$ is the average total number of distinct bonds traversed by (also known as the support) the walk.

If for $t \rightarrow \infty$ asymptotically $B(t) \simeq \beta(t)$, then one may reasonably expect that in that limit $B_{m}(t)$ can be expressed as a scaling function of $m \beta(t) / t$. The sum rules (11) then imply that $B_{m}(t)$ takes the form

$$
B_{m}(t) \simeq \frac{\beta^{2}(t)}{t} \mathcal{B}\left(\frac{m \beta(t)}{t}\right)
$$

where $\mathcal{B}$ is a scaling function.

In this note we will explicit find $\mathcal{B}$ (and $\beta$ ) in spatial dimensions $d=1$ and $d=2$, thereby justifying the scaling form Eq. (2). It appears that $\mathcal{B}$ is an error function "erfc" in $d=1$ and an exponential in $d=2$. In $d=2$ we determine, moreover, the leading order correction to the scaling behavior (2)).

\section{GENERATING FUNCTION METHOD FOR REPEATED BOND TRAVERSALS}

In order to determine $B_{m}(t)$ we need the following notation. Let the vector $\delta$ denote any of the $d$ basis vectors of the lattice. Let $(x, \delta)$ denote the bond between the sites $x$ and $x+\delta$ (the fact that this bond is identical to $(x+\delta,-\delta)$ is of no importance). Let $B_{m}(x, \delta ; t)$ be the probability that $(x, \delta)$ is traversed exactly $m$ times, irrespective of the direction. Then 


$$
B_{m}(t)=\sum_{(x, \delta)} B_{m}(x, \delta ; t)
$$

where each bond occurs exactly once in the summation. The quantity $B_{m}(x, \delta ; t)$ may be calculated by an adaptation of the analogous method for multiple visits to the same site [1]. Let $F(x, \delta ; t)$ be the probability that the first traversal of $(x, \delta)$ occurs at the $t$ th step, with $t=1,2, \ldots$ It is convenient to set $F(x, \delta ; 0) \equiv 0$.

For any $X(t)$ defined for $t=0,1,2, \ldots$ we may introduce the generating function $\hat{X}(z) \equiv \sum_{t=0}^{\infty} z^{t} X(t)$. Let furthermore $R(\tau)$ be the probability that, given a traversal has taken place, the next one occurs exactly $\tau$ steps later, with $\tau=1,2, \ldots$ Then $R(\tau)=F(0, \delta ; \tau)$ and hence $\hat{R}(z)=\hat{F}(0, \delta ; z)$. Reasoning in a similar way as for the site problem one finds that

$$
\hat{B}_{m}(z)=\frac{1}{1-z}\left[\sum_{(x, \delta)} \hat{F}(x, \delta ; z)\right][1-\hat{R}(z)] \hat{R}^{m-1}(z) \quad(m=1,2, \ldots)
$$

Here the function $\hat{F}$, which implies $\hat{R}$, still have to be found.

Let $G(x ; t)$ be the probability that after $t$ steps the walk is at lattice site $x$, for $t=0,1,2, \ldots$ Let $G(x, \delta ; t)$ denote the probability that at its $t$ th step it traverses (in either direction) the bond $(x, \delta)$, for $t=1,2, \ldots$ We set additionally $G(x, \delta ; 0) \equiv 0$. Since $(x, \delta)$ can be traversed starting either from $x$ or from $x+\delta$, we have

$$
G(x, \delta ; t)=\frac{1}{2 d}[G(x ; t-1)+G(x+\delta ; t-1)] \quad(t=1,2, \ldots)
$$

By a slight extension of the standard procedure for calculating first passage probabilities on sites [2, [1. 3] we have here

$$
G(x, \delta ; t)=F(x, \delta ; t)+\sum_{\tau=0}^{t} F(x, \delta ; \tau) G(0, \delta ; t-\tau) \quad(t=1,2, \ldots)
$$

In terms of generating functions, Eqs. (5) and (6) become, respectively, $\hat{G}(x, \delta ; z)=(z / 2 d)[\hat{G}(x ; z)+\hat{G}(x+\delta ; z)]$ and $\hat{F}(x, \delta ; z)=\hat{G}(x, \delta ; z) /[1+\hat{G}(0, \delta ; z)]$. Elimination of $\hat{G}(x, \delta ; z)$ from this pair of equations yields

$$
\hat{F}(x, \delta ; z)=\frac{z}{2 d} \frac{\hat{G}(x ; z)+\hat{G}(x+\delta ; z)}{1+\frac{z}{2 d}[\hat{G}(0 ; z)+\hat{G}(\delta ; z)]}
$$

This achieves the reduction of the desired function $\hat{F}(x, \delta ; z)$ to the known function $\hat{G}(x ; z)$. Note that expression (7) has the required invariance under the replacement $(x, \delta) \rightarrow(x+\delta,-\delta)$. We now exploit the well-known relations $\hat{G}(\delta ; z)=[\hat{G}(0 ; z)-1] / z$ and $\sum_{x} \hat{G}(x ; z)=1 /(1-z)$. Upon combining these with Eqs. (何) and (可) one gets, writing henceforth $G(z) \equiv \hat{G}(0 ; z)$, a fully explicit expression for the generating function $\hat{B}_{m}(z)$ in terms of $G(z)$.

$$
\hat{B}_{m}(z)=\frac{z}{(1-z)^{2}} \frac{(2 d)^{2}}{[2 d+z \tilde{G}(z)]^{2}}\left[\frac{z \tilde{G}(z)}{2 d+z \tilde{G}(z)}\right]^{m-1}
$$

where $\tilde{G}(z)$ is defined by

$$
\tilde{G} \equiv z^{-1}[(1+z) G(z)-1]
$$

The $m$-th coefficient is extracted as

$$
B_{m}(t)=\frac{1}{2 \pi \mathrm{i}} \oint \frac{\mathrm{d} z}{z^{t+1}} \hat{B}_{m}(z)
$$

where the integral runs counterclockwise around the origin. To make $B_{m}(t)$ more explicit, we have to consider each spatial dimension separately. 


\section{ONE DIMENSION}

In dimension $d=1$ we have to evaluate Eq. (9) with [2,1] $G(z)=\left(1-z^{2}\right)^{-1 / 2}$. It turns out to be advantageous to consider the differences $B_{m+1}(t)-B_{m}(t)$. After slight rewriting this yields

$$
B_{m+1}(t)-B_{m}(t)=-\frac{1}{\pi \mathrm{i}} \oint \frac{\mathrm{d} z}{z^{t+m+2}}\left[\sqrt{\frac{1+z}{1-z}}-1\right]\left[1-\sqrt{1-z^{2}}\right]^{m}
$$

We will now fold the integration path around the branch cut that runs from $z=1$ to $z=\infty$ along the positive real axis. In the limit $t \rightarrow \infty$ we expect a meaningful result only if also $m \rightarrow \infty$ at fixed ratio $m / \sqrt{t}$. We anticipate that in this limit the integral on $z$ draws its main contribution from a region at a distance of order $t^{-1 / 2}$ from the branch point at $z=1$. We therefore introduce the scaling variable

$$
\mu_{1}=m / \sqrt{2 t}
$$

and the scaled variable of integration $y=(z-1) t$. The integrand of Eq. (10) may now be expanded in powers of $t^{-1 / 2}$ at fixed $\mu$ and $y$. This leads to

$$
B_{m+1}(t)-B_{m}(t)=-\sqrt{\frac{2}{\pi^{2} t}} \int_{0}^{\infty} \frac{\mathrm{d} y}{\sqrt{y}}\left(\mathrm{e}^{2 \mathrm{i} \mu_{1} \sqrt{y}}+\mathrm{e}^{-2 \mathrm{i} \mu_{1} \sqrt{y}}\right) \mathrm{e}^{-y}
$$

where the two terms on the right hand side come from above and below the branch cut, respectively. The integral is easily found to be equal to $-2^{3 / 2}(\pi t)^{-1 / 2} \mathrm{e}^{-\mu_{1}^{2}}$. With the boundary condition $B_{\infty}(t)=0$ we therefore find upon integrating

$$
B_{m}(t)=2 \operatorname{erfc}\left(\mu_{1}\right)+\mathcal{O}\left(t^{-1 / 2}\right)
$$

which is the final result, valid for $t \rightarrow \infty$ at $\mu_{1}$ fixed. When summing Eq. (13) on $m$ (or alternatively when evaluating the integral obtained by summing Eq. (9) on $m$ ), one obtains $B(t) \simeq \sqrt{8 t / \pi}$. Together with Eq. (13) this confirms the validity of the hypothesized scaling form (2).

In order to compare the large time scaling behavior of $B_{m}(t)$ to its finite time forms we have performed Monte-Carlo simulations. For $t=10^{2}, 10^{3}, 10^{4}$ the bond distribution was averaged over several independent runs. Fig. 1 shows that for increasing values of $t$ the simulation data rapidly converge to the scaling function, and they are practically indistinguishable on this figure for $t \geq 10^{3}$.

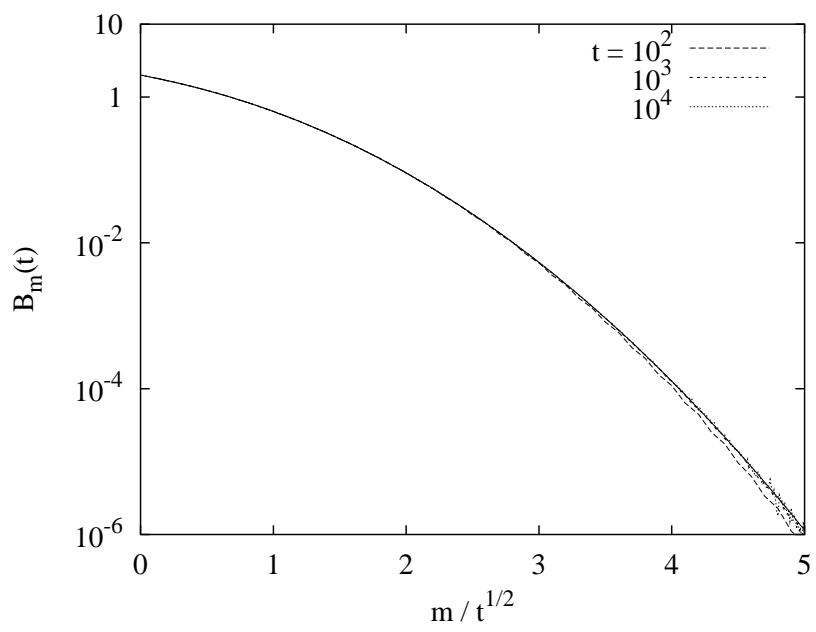

FIG. 1. Simulation results for $B_{m}(t)$ in one dimension at different finite values of time and its scaling form in the $t \rightarrow \infty$ limit (solid line).

\section{TWO DIMENSIONS}

For a two-dimensional square lattice the expansion [2]1] $G(z)=\frac{1}{\pi} \log \frac{8}{1-z}+\mathcal{O}((1-z) \log (1-z))$ enables one to evaluate Eq. (9) for $t$ asymptotically large. The only integral whose asymptotic behavior we need is [4. 3 ] 


$$
\frac{1}{2 \pi \mathrm{i}} \oint \frac{\mathrm{d} z}{z^{t+1}} \frac{1}{(1-z)^{2}} \frac{\pi^{n}}{\log ^{n} \frac{8}{1-z}}=\frac{\pi^{n} t}{\log ^{n} 8 t}\left[1+\frac{n(1-C)}{\log 8 t}+\mathcal{O}\left(\log ^{-2} 8 t\right)\right]
$$

where $C=0.577215 \ldots$ is Euler's constant. We anticipate a meaningful result for $t \rightarrow \infty$ if also $m \rightarrow \infty$ in such a way that the ratio $m / \log t$ remains fixed. We expect that in this scaling limit the $z$ integral will draw its main contribution from a region in the complex plane near the branch point $z=1$ where the ratio $m / G(z)$ is finite. Keeping this in mind we expand the integral (9) for $B_{m}(t)$ as

$$
B_{m}(t)=\frac{1}{2 \pi \mathrm{i}} \oint \frac{\mathrm{d} z}{z^{t+1}} \frac{1}{(1-z)^{2} G^{2}(z)} \mathrm{e}^{-2 m / G(z)}\left[1+\left(\frac{m}{G(z)}-1\right) \frac{1}{G(z)}+\mathcal{O}\left(G^{-2}(z)\right)\right]
$$

We may now insert in (15) the explicit expression $G(z) \simeq \frac{1}{\pi} \log \frac{8}{1-z}$, knowing that the $\mathcal{O}((1-z) \log (1-z))$ terms in the expansion of $G(z)$ will contribute only terms with higher powers of $t^{-1}$ to the final asymptotic series. The exponential in Eq. (15) may then be expanded, the resulting series integrated term by term with the aid of Eq. (14), and summed again. Upon introducing the scaling variable

one finds the final result

$$
\mu_{2}=\frac{2 \pi m}{\log 8 t}
$$

$$
B_{m}(t)=\frac{4 \pi^{2} t}{\log ^{2} 8 t} \mathrm{e}^{-\mu_{2}}\left[1+\left(\mu_{2}-2\right) \frac{\frac{\pi}{2}+1-C}{\log 8 t}+\mathcal{O}\left(\log ^{-2} 8 t\right)\right]
$$

valid for $t \rightarrow \infty$ at $\mu_{2}$ fixed. As expected, the correction terms decay only as powers of the inverse logarithm of the number of steps. The next few higher order terms in the asymptotic series (17) may be calculated without great effort. Since the $k$ th order correction term is multiplied by a $k$ th degree polynomial in $\mu_{2}$, this is actually an expansion in powers of $\mu_{2} / \log 8 t$. One may verify that Eq. (17) satisfies the sum rules (11) up to and including the first order correction term. The scaling function $\mathcal{B}$ introduced in Eq. (2) here appears to be a simple exponential. One finds that the average total number of bonds traversed increases as $\beta(t)=2 \pi t / \log 8 t+\mathcal{O}\left(t \log ^{-2} 8 t\right)$.

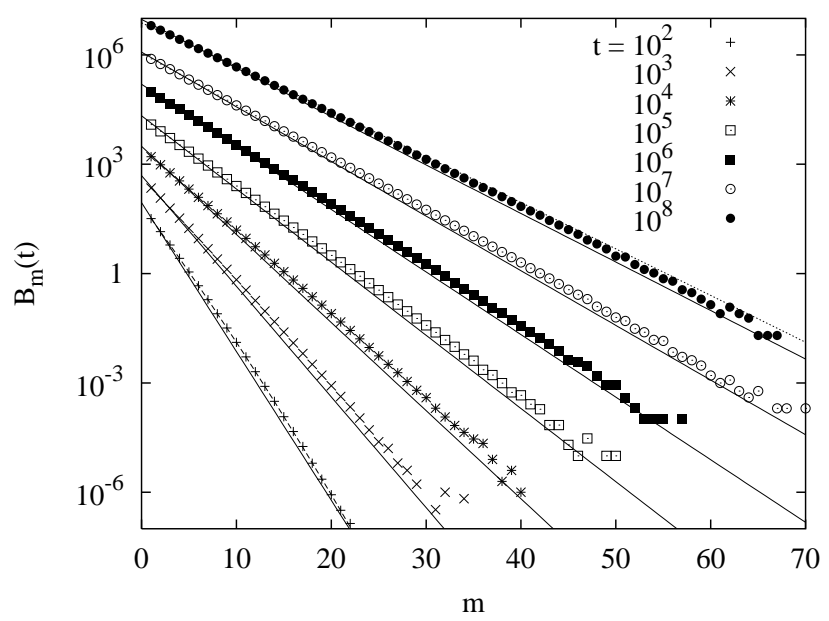

FIG. 2. Simulation data for $B_{m}(t)$ in two dimensions at different values of time (symbols) compared to the theoretical scaling form (solid lines). The exact result for $t=10^{2}$ (dashed line) and the scaling law plus leading correction for $t=10^{8}$ (dotted line) are also displayed.

Fig. 2 compares simulation data for $t=10^{2}, 10^{3}, \ldots, 10^{8}$ to the theoretical scaling law (the first term of Eq. (177)). The $t=10^{2}$ data are also compared to the exact curve, which we obtained by Taylor expanding Eq. (8) through the one hundredth term with the aid of a symbol manipulation program. For $t=10^{8}$ the scaling law plus leading correction (the first two terms of Eq. (177) ) is also displayed. Upon collapsing the data of Fig. 2 one finds Fig. 3, which shows that in $d=2$ the convergence to the asymptotic scaling law is very slow. 


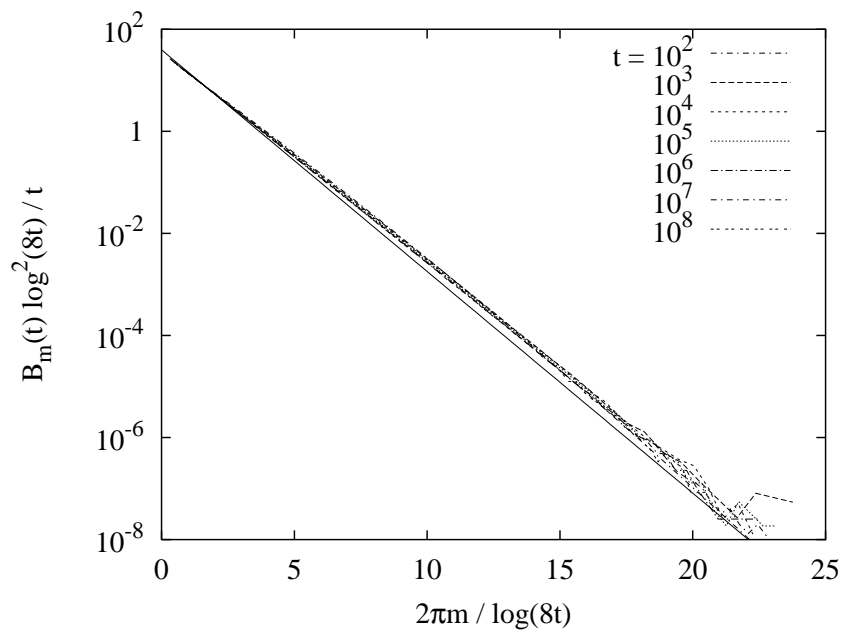

FIG. 3. Data collapse of two dimensional simulation results for $B_{m}(t)$. The $t \rightarrow \infty$ scaling limit is shown as a solid line.

\section{CONCLUDING REMARKS}

We conclude this note with a series of comments and remarks.

An alternative route to $B_{m}(t)$ is to consider a complementary process, by focusing on a particular bond in, say, a finite periodic $L^{d}$ lattice. Now, we can ask how often this bond is traversed by a $t$-step random walk which starts at $x_{0}$ and ends at $x$. By summing over $\left(x, x_{0}\right)$ and invoking translational invariance, we obviously access $B_{m}(t)$. In this approach, we can easily generalize the problem to a study of the frequency of traversing in only one direction. To solve both problems, consider a modified random walker, for which the rate of traversing our chosen bond is $p / 2 d$ instead of $1 / 2 d$. Solving the master equation for $P\left(x, t \mid x_{0}, 0 ; p\right)$ (the usual probability) by standard generating function techniques and defining $\tilde{B}(p, z) \equiv \sum_{x, x_{0}} \sum_{t=0}^{\infty} z^{t} P\left(x, t \mid x_{0}, 0 ; p\right)$, we obtain

$$
\tilde{B}(p, z)=\frac{L^{d}}{1-z}-\frac{2 z(1-p)}{(1-z)^{2}[2 d+(1-p) z \tilde{G}(z)]} .
$$

The first term represents, since $\sum_{x} P\left(x, t \mid x_{0}, 0 ; p=1\right)=1$, the $L^{d}$ points of origination of the walks we considered. Due to this difference in normalization, the bivariate generating function for $B_{m}(t)$, i.e. $\sum_{m=1}^{\infty} \sum_{t=0}^{\infty} p^{m} z^{t} B_{m}(t)$, is precisely $d[\tilde{B}(p, z)-\tilde{B}(0, z)]$. With the extra factor $d$, the quantity $d \tilde{B}(1, z)$ represents nothing but the total number of bonds in the lattice. Interestingly, this $\tilde{B}(p, z)$ contains an extra term $(\tilde{B}(0, z))$ which carries the information on the bonds never traversed. So, $d\left[L^{d} /(1-z)-\tilde{B}(0, z)\right]$ is just $\sum_{t=0}^{\infty} z^{t} B(t)$. For completeness, we report the result for unidirectional traverses:

$$
\tilde{B}(p, z)=\frac{L^{d}}{1-z}-\frac{z(1-p)}{(1-z)^{2}[2 d+(1-p) z G(z)]} .
$$

Finally, note that finite size effects are fully incorporated in this approach, although they are implicitly "buried" in $G(z)=L^{-d} \sum_{\left\{k_{i}\right\}}\left[1-(z / d) \sum_{i=1}^{d} \cos k_{i}\right]^{-1}$ (where the sum is over the set $\left\{k_{i}\right\}$ of allowed $L^{d}$ wavevectors). Needless to say, generalizations to strip- or slab-like samples $\left(L_{1} \leq L_{2} \leq \ldots\right)$ are straightforward. Thus, it is possible to study the crossover of our distributions, at least in principle, when the polymer length exceeds the shortest dimension significantly $\left(t \gg L_{1}\right)$. Physically, ultra-thin membranes made from extra-long polymers can be manufactured. Until they become reality, however, it may not be worthwhile to extend our analysis to this class of crossover behavior.

A second remark concerns the statistics of multiple visits to sites, as opposed to traversals of bonds. Visits have been of considerable interest in the literature (see Hughes [1] and references cited therein). The average number $V_{m}(t)$ of sites visited exactly $m$ times by a $t$ step random walk was studied by Montroll and Weiss [5] and by Barber and Ninham [6]. The $t \rightarrow \infty$ limit at fixed $m$ was considered by Hughes [1] (see also [7]) in $d=1$ and by Montroll and Weiss [5] (see also 8]) in $d=2$; however, the scaling limit expression of $V_{m}(t)$ has not to our knowledge appeared in 
the literature. Since the analysis of $V_{m}(t)$ runs exactly parallel to that of $B_{m}(t)$, we content ourselves to state the results here.

In dimension $d=1$ the average number of sites visited $m$ times is, to leading order in the scaling limit, equal to the average number of bonds visited $m$ times: $V_{m}(t)=2 \operatorname{erfc}\left(\mu_{1}\right)$ for $t \rightarrow \infty$ at $\mu_{1}$ fixed. In dimension $d=2$ one has in terms of the scaling variable $\mu_{2}^{\prime}=\pi m / \log 8 t$

$$
V_{m}(t)=\frac{\pi^{2} t}{\log ^{2} 8 t} \mathrm{e}^{-\mu_{2}^{\prime}}\left[1+\left(\mu_{2}^{\prime}-2\right) \frac{\frac{\pi}{2}-1+C}{\log 8 t}+\mathcal{O}\left(\log ^{-2} 8 t\right)\right]
$$

valid for $t \rightarrow \infty$ at $\mu_{2}^{\prime}$ fixed. Comparison of Eqs. (19) and (17) shows that to leading order $V_{m}(t)$ and $B_{m}(t)$ are identical up to a coefficient and a scale factor; however, the coefficients of the first correction terms are different. The leading order relation between $V_{m}(t)$ and $B_{m}(t)$ in $d=1,2$ is heuristically clear as follows. Given a large number $m$ of visits to an arbitrary site $x$, there will have been typically $m /(2 d)$ traversals, starting from $x$, of a specific bond $(x, x+\delta)$. Now the same bond will have been traversed, typically, the same number of times in the opposite direction. So for each site visited $m$ times, there are $d$ bonds traversed $m / d$ times.

Although we have not shown so explicitly, the scaling function in $d=2$ is expected to be universal, i.e. lattice structure independent. In dimensions $d>2$ the random walk is transient and it is easy to show that as a consequence in the large $t$ limit $B_{m}(t) \simeq b_{m} t$ and $V_{m}(t) \simeq v_{m} t$, where $b_{m}$ and $v_{m}$ are nonuniversal. For random walks that are not simple (i.e. have a step size distribution not limited to nearest neighbor steps), bond traversals are not unambiguously defined. However, visits to sites still are, and for such walks $V_{m}(t)$ is readily calculated by the present method. One case of interest is the scaling limit of $V_{m}(t)$ for lattice Lévy flights (also called Riemann walks) [9, 10], for which the distribution of step sizes decreases as a power law.

\section{ACKNOWLEDGMENTS}

We thank J. Das, Manoj Gopalakrishnan, B. Schmittmann, and U.C. Tauber for helpful discussions. T.A. and H.J.H. acknowledge the warm hospitality of the Physics Department of Virginia Tech, where part of this research was conducted. This research was supported in part by grants from the Swiss National Science Foundation, the Hungarian Academy of Sciences (Grant No. OTKA T029792), and the US National Science Foundation through the Division of Materials Research. The Laboratoire de Physique Théorique in Orsay is associated with Centre National de la Recherche Scientifique as research unit UMR8627.

[1] B.D. Hughes, Random Walks and Random Environments, Vol. 1: Random Walks (Clarendon Press, Oxford 1996).

[2] G.H. Weiss, Aspects and Applications of the Random Walk (North-Holland, Amsterdam 1994).

[3] F. van Wijland and H.J. Hilhorst, J. Phys. A 30 (1997) 507.

[4] F.S. Henyey and V. Seshadri, J. Chem. Phys. 76 (1982) 5530.

[5] E.W. Montroll and G.H. Weiss, J. Math. Phys. 6 (1965) 167.

[6] M.N. Barber and B.W. Ninham, Random and Restricted Walks: Theory and Applications (Gordon and Breach, New York 1970).

[7] D. Newman, SIAM Review 26 (1984) 573.

[8] P. Erdös and S.J. Taylor, Acta Mathematica Academiae Scientiarum Hungaricae 11 (1960) 137.

[9] J.E. Gillis and G. Weiss, J. Math. Phys 11 (1970) 1307.

[10] A.M. Mariz, F. van Wijland, H.J. Hilhorst, S.R. Gomes Júnior, and C. Tsallis, J. Stat. Phys. 102 (2001) 259. 